\title{
Serum C-reactive protein and metabolic components
}

\author{
Tomoyuki Kawada
}

Received: 5 April 2013/Accepted: 17 April 2013/Published online: 26 April 2013

(C) Springer Science+Business Media New York 2013

I read with interest the recent contribution by Yang et al. [1], in Endocrine. The same content was also reported by Mahajan et al. [2], by including markers of insulin resistance. Both studies adopted a cross-sectional design, and causal relationship should be explored by longitudinal study. As a common finding, obesity was strongly related to serum C-reactive protein (CRP) compared with other metabolic components. In contrast, hyperglycemia and elevated blood pressure were significant, but relatively weak association with CRP.

I have some concerns on study by Yang et al. [1]. First, they used sex as adjusting variable for the total group of metabolic syndrome (MetS) and for each metabolic component. The odds ratio (95\% confidence interval) of the highest tertile of CRP for MetS is 5.04 (3.41-7.45) in men and $9.92(6.64-14.82)$ in women. As there is a sex difference of odds ratio, stratified analysis by sex is recommended in every analysis.

Second, Yang et al. and Mahajan et al. included patients associated with MetS, respectively. On this point, the distribution in the number of MetS by Yang et al. skews left compared by the distribution by Mahajan et al., namely data by Yang et al., includes relatively more subclinical subjects in their study compared with data by Mahajan et al. Risk assessment should be conducted by understanding the characteristics of the target subjects.

Third, diabetes mellitus is a risk factor for atherosclerotic cardiovascular disease, including coronary heart disease and ischemic stroke. In addition, there is a report that elevated
CRP was independently associated with risk of impaired fasting glucose and/or impaired glucose tolerance [3]. This means that subclinical association between CRP and several metabolic components should also be evaluated. The odds ratios (95\% confidence intervals) of the highest group of CRP for hyperglycemia are $2.10(1.56-2.84)$ and 1.27 (101-1.59) by Yang et al. and Mahajan et al., respectively. The outcome concerning the relationship between CRP and hyperglycemia should be evaluated in other ethnic groups to keep generalizability of the study.

Disclosure statement The author has indicated no financial support.

Conflict of interest There is no conflict of interest in this study.

\section{References}

1. T. Yang, C.H. Chu, P.C. Hsieh, C.H. Hsu, Y.C. Chou, S.H. Yang, C.H. Bai, S.L. You, L.C. Hwang, T.C. Chung, C.A. Sun, C-reactive protein concentration as a significant correlate for metabolic syndrome: a Chinese population-based study. Endocrine 43, 351-359 (2013)

2. A. Mahajan, A. Jaiswal, R. Tabassum, A. Podder, S. Ghosh, S.V. Madhu, S.K. Mathur, N. Tandon, D. Bharadwaj, Elevated levels of C-reactive protein as a risk factor for metabolic syndrome in Indians. Atherosclerosis 220, 275-281 (2012)

3. A. Jaiswal, R. Tabassum, A. Podder, S. Ghosh, N. Tandon, D. Bharadwaj, Elevated level of C-reactive protein is associated with risk of prediabetes in Indians. Atherosclerosis 222, 495-501 (2012)
T. Kawada $(\bowtie)$

Department of Hygiene and Public Health, Nippon Medical School, 1-1-5 Sendagi, Bunkyo-Ku, Tokyo 113-8602, Japan e-mail: kawada@nms.ac.jp 